\title{
Short-term changes in cerebral activity in on-pump and off-pump cardiac surgery defined by functional magnetic resonance imaging and their relationship to microembolization
}

\author{
Yasir Abu-Omar, MBChB, MRCS, a,b Sarah Cader, MRCP, ${ }^{\mathrm{b}}$ Lorenzo Guerrieri Wolf, MD, ${ }^{\text {a }}$ David Pigott, FRCA, \\ Paul M. Matthews, MD, DPhil, FRCP, and David P. Taggart, MD, PhD, FRCS
}

From the Department of Cardiothoracic Surgery, John Radcliffe Hospital, Oxford, UK, ${ }^{a}$ and the Department of Clinical Neurology, Centre for Functional Magnetic Resonance Imaging of the Brain, University of Oxford, Oxford, UK. ${ }^{\mathrm{b}}$

Supported by the British Heart Foundation. Received for publication Oct 23, 2005; revisions received April 14, 2006; accepted for publication April 24, 2006.

Address for reprints: David P. Taggart, MD, PhD, FRCS, Department of Cardiothoracic Surgery, John Radcliffe Hospital, Oxford, OX3 9DU, UK (E-mail: yabuomar@ doctors.org.uk).

J Thorac Cardiovasc Surg 2006;132:1119-25 0022-5223/ $\$ 32.00$

Copyright () 2006 by The American Association for Thoracic Surgery

doi:10.1016/j.jtcvs.2006.04.057
Objective: Cognitive dysfunction is common early after cardiac surgery. We previously reported that functional magnetic resonance imaging of the brain can detect subclinical changes in prefrontal cortical activation after coronary artery bypass grafting. In this study, we used functional magnetic resonance imaging to contrast perioperative prefrontal activation in patients undergoing on-pump and off-pump coronary artery bypass grafting and to relate differences to cerebral microembolic load.

Methods: Functional images of the brain were acquired in 25 patients undergoing cardiac surgery ( 13 off-pump and 12 on-pump) before surgery and 4 weeks after surgery during performance of a verbal memory task of increasing complexity (n-back task). Continuous intraoperative transcranial Doppler scanning was performed to quantify the number of cerebral microemboli. Perioperative changes in task-associated prefrontal activation were compared between the 2 groups and were then correlated with the number of microemboli recorded during surgery.

Results: The median (interquartile range) number of detected microemboli was 35 (21-63) in the off-pump group and 254 (116-397) in the on-pump group $(P<.005)$. Functional imaging performed before surgery demonstrated increased activity in the prefrontal regions with increasing task complexity. After surgery, there was a significant reduction in task-associated prefrontal activation in the on-pump, but not in the off-pump, group $(P<.05)$. There was a negative correlation between the perioperative signal changes in the prefrontal region and the total number of microemboli $(r=-0.63 ; P<.01)$.

Conclusions: Patients undergoing on-pump, but not off-pump, surgery have a significant relative reduction in prefrontal activation, which correlates with intraoperative cerebral microembolic load. We hypothesize that this reduction in activation is related to subclinical functional impairments and that microembolic load is an important mechanism of perioperative cerebral insult.

$\mathrm{N}$ eurologic injury is one of the most important complications of otherwise successful coronary artery bypass grafting $(\mathrm{CABG}) .{ }^{1}$ Although overt injury such as stroke affects only a small percentage of patients, cognitive impairment is detectable in approximately half of patients early after surgery, in approximately a quarter at 6 months, and in $40 \%$ at 5 years. ${ }^{2,3}$ Of more concern, its occurrence early after surgery correlates both with later decline in cognitive function and with an impaired quality of life. ${ }^{3,4}$ Microembolism is probably the most important pathophysiological mechanism, although intraoperative cerebral hypoper- 


\section{Abbreviations and Acronyms \\ $\mathrm{CABG}=$ coronary artery bypass grafting \\ $\mathrm{CPB}=$ cardiopulmonary bypass \\ fMRI = functional magnetic resonance imaging \\ ONCABG $=$ on-pump coronary artery bypass grafting \\ $\mathrm{OPCABG}=$ off-pump coronary artery bypass grafting}

fusion and the systemic inflammatory response syndrome have also been implicated. ${ }^{1,5}$

In a preliminary study of 16 patients undergoing onpump, off-pump, and open cardiac surgery, we reported postoperative changes in functional magnetic resonance imaging (fMRI) activity in a localized region of the prefrontal cortex during performance of a simple memory task. ${ }^{6}$ Furthermore, our data suggested that these changes might be related to the number of intraoperative microemboli. ${ }^{6}$ On the basis of our preliminary study and the fact that global prefrontal activity is impaired in certain pathologic states such as frontotemporal dementia, ${ }^{7}$ we hypothesized that similar global changes might be detected in a larger cohort of patients undergoing cardiac surgery and that the changes might be related to microembolic load.

\section{Materials and Methods Patients}

Ethical approval for the study was obtained from the Central Oxford Research Ethics Committee (OxREC C01.258), and all patients gave informed consent. Twenty-five patients undergoing CABG were recruited for the study. Twelve patients had on-pump CABG (ONCABG), and 13 had off-pump surgery (OPCABG). Of the 25 patients described here, 12 had been previously included in a preliminary analysis of postoperative superior and middle frontal gyral activity - an area known to be involved in verbal memory - as a region of interest by using a simple cognitive task (1-back task). ${ }^{6}$ The purpose of that study was to explore the ability to assess postoperative cerebral injury in a heterogeneous group of patients undergoing different cardiac surgical procedures using fMRI techniques as a pilot to this study. All patients in this study underwent structural and functional MRI of the brain (during performance of a full n-back task with increasing complexity level) before surgery and 4 to 6 weeks after surgery. Functional MRI activation patterns were assessed by using more sophisticated assessments of memory to allow for task-specific activations to be studied during performance of a full n-back task with an increasing complexity level (0-back to 3-back) in patients undergoing ONCABG and OPCABG. In addition, the patients had intraoperative continuous bilateral transcranial Doppler monitoring.

\section{Cardiopulmonary Bypass}

After full anticoagulation with heparin given at a dose of 300 IU/kg to maintain an activated clotting time of 400 to 600 seconds, cardiopulmonary bypass (CPB) was instituted by using ascending aortic cannulation and a 2 -stage right atrial venous cannulation. A roller pump (Jostra HL 20, Hirlingen, Germany) and hollow-fiber membrane oxygenator (Affinity NT; Medtronic Inc, Minneapolis, Minn) were used. The extracorporeal circuit was primed with 1000 $\mathrm{mL}$ of Hartmann solution and $2500 \mathrm{IU}$ of heparin. CPB was maintained with nonpulsatile flow with a flow rate of $2.4 \mathrm{~L} \cdot \mathrm{m}^{-2}$ - $\min ^{-1}$ at normothermia; the temperature was allowed to drift to $34^{\circ} \mathrm{C}$. Acid-base balance was managed with alpha-stat control. Myocardial protection was achieved with intermittent antegrade cold crystalloid cardioplegia. Cardiotomy suction was used. On completion of all distal anastomoses, the aortic crossclamp was removed, and each proximal anastomosis was performed with individual application of a partial aortic side clamp onto palpably normal aorta; epiaortic ultrasonography was not routinely used. Arterial line filtration was used in the last 5 patients in the ONCABG group after a change in our practice, in which filters became routinely used during $\mathrm{CPB}$.

\section{Off-pump CABG}

For OPCABG, complete anticoagulation with heparin was achieved as in the CPB group. Regional myocardial immobilization was achieved with a suction stabilizer (Octopus [Medtronic Inc, Minneapolis, Minn] or Guidant [Guidant Corporation, Santa Clara, Calif]). The target coronary vessels were snared proximally with a silicone rubber sling. An intracoronary shunt (Guidant Axius) was used only when there was hemodynamic compromise during construction of the anastomosis (usually the distal right coronary artery). Visualization was enhanced by using a surgical blower-mister device (Medtronic Clearview; Medtronic Inc). Where possible, patients had composite arterial grafts, with complete avoidance of aortic manipulation.

\section{Transcranial Doppler}

Continuous intraoperative monitoring was performed by using a multifrequency Doppler system (Embodop, DWL Electronische Systeme GmbH, Singen, Germany), by using a protocol that we have previously described. ${ }^{8}$ Briefly, dual-frequency (2.0- and 2.5$\mathrm{MHz}$ ) probes were used to simultaneously insonate transtemporal windows over both middle cerebral arteries by using a specifically designed head brace. The middle cerebral artery insonation depth was set between 45 and $55 \mathrm{~mm}$, with a sample volume of $13 \mathrm{~mm}$. An additional 2.0-MHz insonation reference gate was set $15 \mathrm{~mm}$ superficial to the middle cerebral artery insonation gate. This reference gate serves for the online rejection of artifacts, because these are identified when high-intensity transient signals are detected in both gates (middle cerebral artery and reference gates) simultaneously or with a time delay of less than 4 milliseconds. ${ }^{9}$ This multifrequency system also differentiates between solid and gaseous microemboli, because solid microemboli reflect more ultrasound at higher than at lower frequencies, whereas the opposite occurs in the case of gaseous microemboli. ${ }^{10}$ The differentiation occurs online during monitoring, and the data are recorded on a computer hard drive, thus allowing off-line analysis to be performed.

\section{MRI Image Acquisition}

A 3.0-T (Varian Inova, Lake Forest, Calif) scanner with a quadrature birdcage radiofrequency head coil was used. An echo-planar imaging sequence (24 slices; repetition time, 3 seconds; echo time, 30 milliseconds; field of view, $192 \times 256 \mathrm{~mm}$; matrix, $64 \times 64$ ) 
was used to acquire fMRI data during performance of a verbal memory paradigm. Structural T1-weighted (3D Turbo FLASH sequence; repetition time, 15 seconds; echo time, 6.9 milliseconds) images were obtained.

\section{Verbal Memory Paradigm and Stimulus Presentation}

A verbal memory paradigm of increasing task complexity was used: a n-back task including 0-, 1-, 2-, and 3-back in pseudorandomized block arrangements. ${ }^{11}$ Briefly, in the 0-back (control) task, a target letter is presented at the start of the task, and in the remainder of the tasks, the patient attempts to identify letters seen 1,2 , or 3 letters back in the series. ${ }^{11}$

\section{Functional MRI Image Analysis}

Image analysis to reveal significant brain activity based on changes in blood oxygenation level dependent (BOLD) signal was performed on each patient's data by using FEAT (FMRIB Easy Analysis Tool; http://www.fmrib.ox.ac.uk/fsl/). ${ }^{12}$ Analyses were performed by an observer blinded to the nature of surgical intervention (Y.A.-O.). These were cross-examined by a second independent observer (S.C.). Before statistical analysis, the following processing was applied: motion correction using MCFLIRT, ${ }^{13,14}$ spatial smoothing using a gaussian kernel of full width at half maximum $5 \mathrm{~mm}$ and nonlinear high-pass temporal filtering with a high-pass filter cutoff of 100 seconds. The fMRI signal was then modeled by using general linear modeling. Individual contrasts measured fMRI activation during 1-, 2-, and 3-back tasks relative to the 0-back (control) task. Group contrasts were generated with a mixed-effects model, which included contributions from individual measurement variance and variance between individuals within the groups. Region-of-interest analyses were performed to assess changes in activity with increasing task complexity. This measured mean signal intensity change within the region of interest before and after surgery.

\section{Statistical Analysis and Presentation of Data}

Patient characteristics are presented as mean \pm SD. Normally distributed data were compared by using the Student $t$ test, and categorical variables were compared by using the $\chi^{2}$ test. The number of microemboli is presented as the median and interquartile range, and the nonparametric Mann-Whitney $U$ test was used to compare the difference in microembolization between the ON$\mathrm{CABG}$ and OPCABG groups. The differences in performance were compared by using analysis of variance. Activations in the prefrontal cortex were compared between the 2 groups at each complexity level by using repeated-measures analysis of variance. All statistical analyses were performed by using SPSS for Windows, version 11.5 (SPSS Inc, Chicago, Ill).

\section{Results}

Patient characteristics are summarized in Table 1, and both groups were similar in terms of age, sex, left ventricular function, risk scores, and number of grafts. No patient showed clinical evidence of stroke, and no changes consistent with ischemia were seen in comparisons of preoperative and postoperative T1-weighted MRI scans. The median
TABLE 1. Perioperative characteristics

\begin{tabular}{lccc}
\hline Variable & OPCABG & ONCABG & $P$ value \\
\hline $\mathrm{n}$ & 13 & 12 & - \\
Age, y (mean \pm SD) & $60 \pm 8$ & $59 \pm 10$ & .77 \\
No. female & 2 & 1 & .59 \\
Impaired LV function, n (\%) & $4(31 \%)$ & $4(33 \%)$ & .89 \\
Parsonnet score (mean \pm SD) & $4.7 \pm 4.9$ & $4.5 \pm 4.7$ & .27 \\
No. grafts (mean \pm SD) & $2.5 \pm 0.7$ & $2.9 \pm 0.8$ & .25
\end{tabular}

OPCABG, Off-pump coronary artery bypass grafting; ONCABG, on-pump coronary artery bypass grafting; $L V$, left ventricular.

follow-up in the OPCABG and ONCABG groups was 37 and 44 days, respectively.

\section{Microembolism}

The median (interquartile range) number of detected microemboli was 35 (21-63) in the OPCABG group and 254 $(116-397)$ in the ONCABG group $(P<.005)$. Solid microemboli accounted for $15 \%$ and $25 \%$ of the total number of microemboli in the OPCABG and ONCABG groups, respectively.

\section{Verbal Memory Data}

Performance on the verbal memory task is illustrated in Figure 1. There were no differences between preoperative and postoperative task scores between the patient groups. The median (interquartile range) overall score in the ONCABG group was 77\% (56\%-94\%) before surgery and $78 \%(64 \%-88 \%)$ after surgery $(P=.95)$. In the off-pump group, the respective scores were $82 \%$ (70\%-100\%) before surgery and $82 \%(66 \%-95 \%)$ after surgery $(P=.44)$, respectively.

\section{Functional Imaging}

Before surgery, the verbal memory task was associated with significant activation in several brain regions, including the prefrontal, posterior parietal, and anterior cingulate area, as has been reported previously. ${ }^{15}$ There was a monotonic increase in activation in these regions with increasing task complexity (Figure 2). On the basis of our a priori hypothesis, we tested for differences in perioperative changes in activity in the prefrontal activation region of interest between the 2 groups. We found a significant reduction in activation after surgery in the ONCABG patients compared with the OPCABG group $(P<.05$; Figures 3 and 4$)$.

\section{Microembolism and Postoperative Cerebral Activity} To understand why fMRI differences were observed between the OPCABG and ONCABG groups, we tested for the relationship between activation changes and intraoperative embolic load. Perioperative changes in prefrontal 


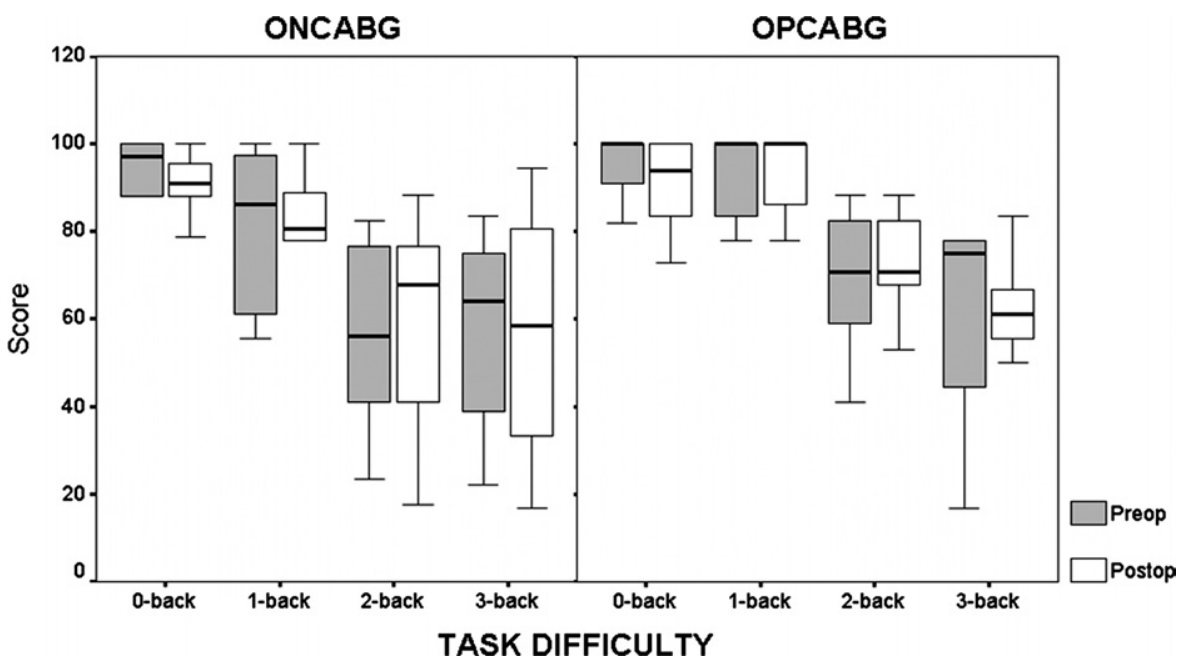

Figure 1. Comparison of preoperative and postoperative scores in the n-back tasks before (Preop) and after (Postop) surgery in the ONCABG and OPCABG groups.

cerebral activity showed a significant negative correlation with the total number of microemboli recorded during surgery $(r=-0.63$; corrected $P<.01)$ for the 3-back task (when the highest task-related activation occurs)

\section{Discussion}

To our knowledge, MRI has not been used previously to specifically explore the effect of ONCABG and OPCABG on postoperative cerebral function. This study confirms and extends the findings of our previous study, in which we reported a nonspecific overall reduction in postoperative cerebral activity and a potential correlation between the postoperative activation pattern and intraoperative microembolization. ${ }^{6}$ There were 2 key findings from this work. First, although there was no apparent difference in postoperative verbal memory scores between the 2 groups, there was a relative reduction in fMRI signal intensity in ONCABG patients in the prefrontal region, thus suggesting greater neuronal dysfunction. The second main finding was that this reduction in fMRI signal intensity change correlated with the number of microemboli detected during surgery.

\section{Relevance of Signal Intensity Change}

The normal pattern of response in this brain region is a monotonic increase in fMRI activation with increasing memory load. ${ }^{11,16}$ Relative reductions in prefrontal activation with memory tasks occur in early frontotemporal dementia or Alzheimer disease. ${ }^{7,17}$ In our study, both groups showed a normal pattern of activity before surgery, but although this was maintained in the OPCABG group at 1 month after surgery, it was lost in the ONCABG group. Whether this is due to reduced neuronal activity and information processing in the prefrontal cortex or an altered balance of excitatory and inhibitory inputs is not clear from our study. ${ }^{18}$

Because this study was limited to a single postoperative time point of 4 to 6 weeks after surgery, we do not know how long depression of prefrontal activity in ONCABG patients persists. This warrants further investigation, because 5-year follow-up studies of on-pump cardiac surgery patients demonstrated a significant decline in neurocognitive function that correlated with the severity of the initial perioperative insult. ${ }^{3}$

\section{Lack of Correlation Between Verbal Memory Score and Signal Intensity Change}

Despite a difference in fMRI signal intensity change, there was no significant postoperative change in verbal memory scores in either group. A possible explanation is that the patient numbers were too few to detect meaningful differences in cognitive behavioral scores given the interindividual variances expected with these measures. Indeed, we have argued that, because of their sensitivity to both internal and external influences in the postoperative period, the noise-to-signal ratio may be too high to detect subtle but real changes in cognitive function. ${ }^{19}$ In their detailed review, Wilkinson and Halligan ${ }^{20}$ emphasized the limitations of behavioral scores and suggested that fMRI may be substantially more sensitive to functional differences.

Another possibility, however, is that alternative brain areas may functionally compensate for prefrontal impairment, thus allowing preservation of clinical test performance. There is an increasing appreciation in other contexts for the role of neural plasticity, whereby an alternative region of the brain can increase activity to compensate for decreased activity in a damaged area. ${ }^{21,22}$ This is seen, for example, in motor recovery after stroke, where activity in 


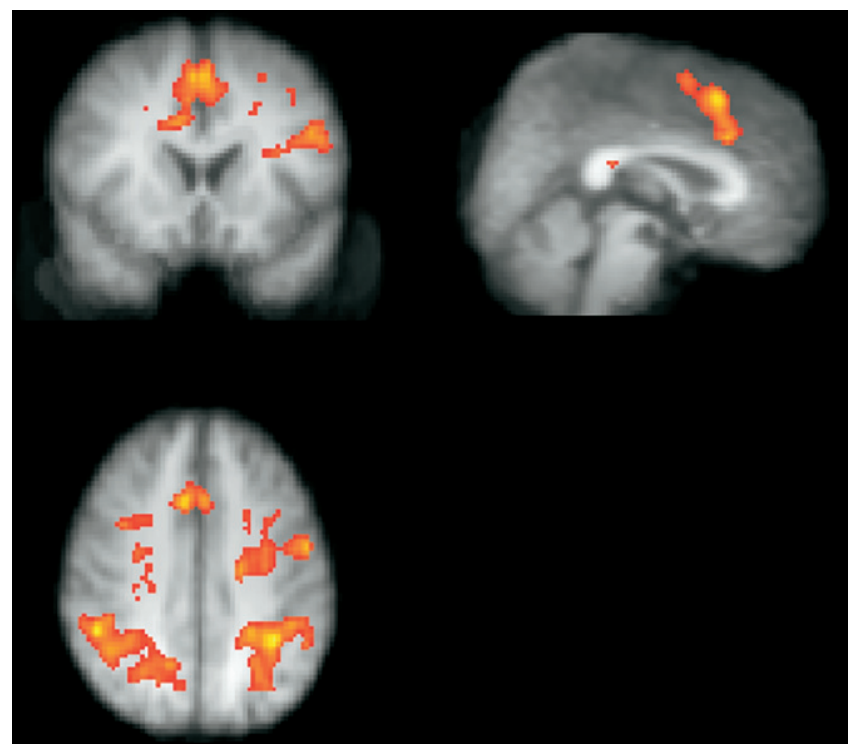

Figure 2. Preoperative fMRI activation in 25 patients during performance of verbal memory task. The images show areas with an increase in activity with increasing task complexity. Activations are seen in the prefrontal and posterior parietal regions bilaterally and in the anterior cingulate area. $F M R I$, functional magnetic resonance imaging.

motor areas in the unaffected hemisphere may adaptively compensate for damaged regions. ${ }^{21}$

\section{Role of Microembolization}

Our results suggest that the difference in fMRI signal intensity change between ONCABG and OPCABG patients may be due to the greater degree of cerebral microembolism in the former. Cerebral microemboli are strongly implicated in the pathogenesis of cognitive decline after $\mathrm{CPB},{ }^{23-25}$ and large numbers of lipid microemboli have been documented in the brains of patients dying after cardiac surgery, thus demonstrating the potential pathologic importance of intraoperative microembolism. ${ }^{26}$ An important source of particulate microemboli is the ascending aorta, especially after manipulation, ${ }^{8}$ whereas lipid microemboli may result from the use of cardiotomy suction and denaturation of proteins. ${ }^{27}$ It has therefore been proposed that avoidance of cardiotomy suction can result in a significant reduction in lipid microemboli.

Recently, using new transcranial Doppler ultrasonography techniques, we reported quantitative and qualitative differences in microembolization in patients undergoing on-pump compared with off-pump surgery. ${ }^{8}$ We also reported preliminary evidence of a correlation between intraoperative microemboli and postoperative cerebral activation patterns during performance of a related cognitive task in patients undergoing $\mathrm{CABG}$ and open cardiac surgery. ${ }^{6}$ In

\section{(a) OPCABG}

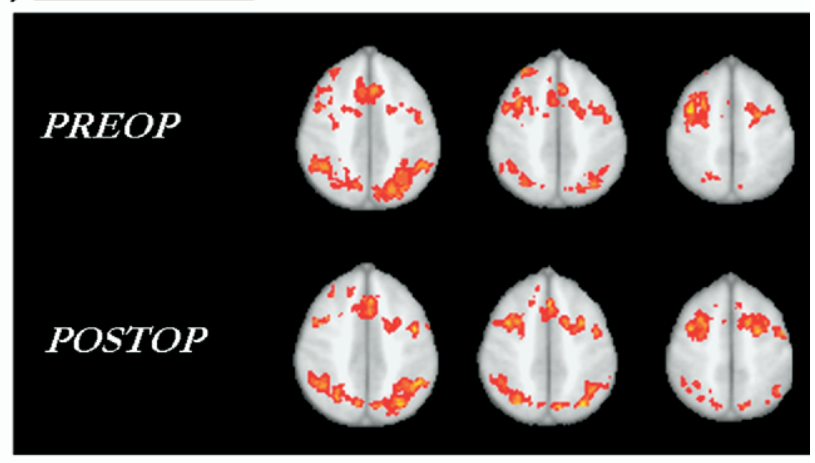

(b) ONCABG



Figure 3. Mean group activation images in the 2 groups. These show no postoperative difference in the activation pattern in patients undergoing off-pump surgery but show a significant reduction in activation in the prefrontal regions after surgery in the on-pump group (arrows). Preop, Before surgery; postop, after surgery.

this study, the perioperative changes in prefrontal activation were inversely correlated with the number of intraoperative microemboli, thus indicating that increasing numbers of microemboli resulted in increasing changes in functional activity; this may represent functional impairment.

\section{Clinical Implications of Our Findings}

OPCABG is already established as a safe and effective procedure, and its major benefits are likely to be seen in higher-risk patients. ${ }^{28}$ Our findings are particularly important because we chose to study a relatively young and low-risk population of patients who are traditionally considered to be at the least risk of cognitive dysfunction. It could therefore be anticipated that an older population, who are at greater risk of cerebral injury, might demonstrate more profound changes.

This study offers new insights into cerebral dysfunction after cardiac surgery. Functional MRI may be more sensitive than neuropsychological testing. By providing additional information on localization of change, it may offer 

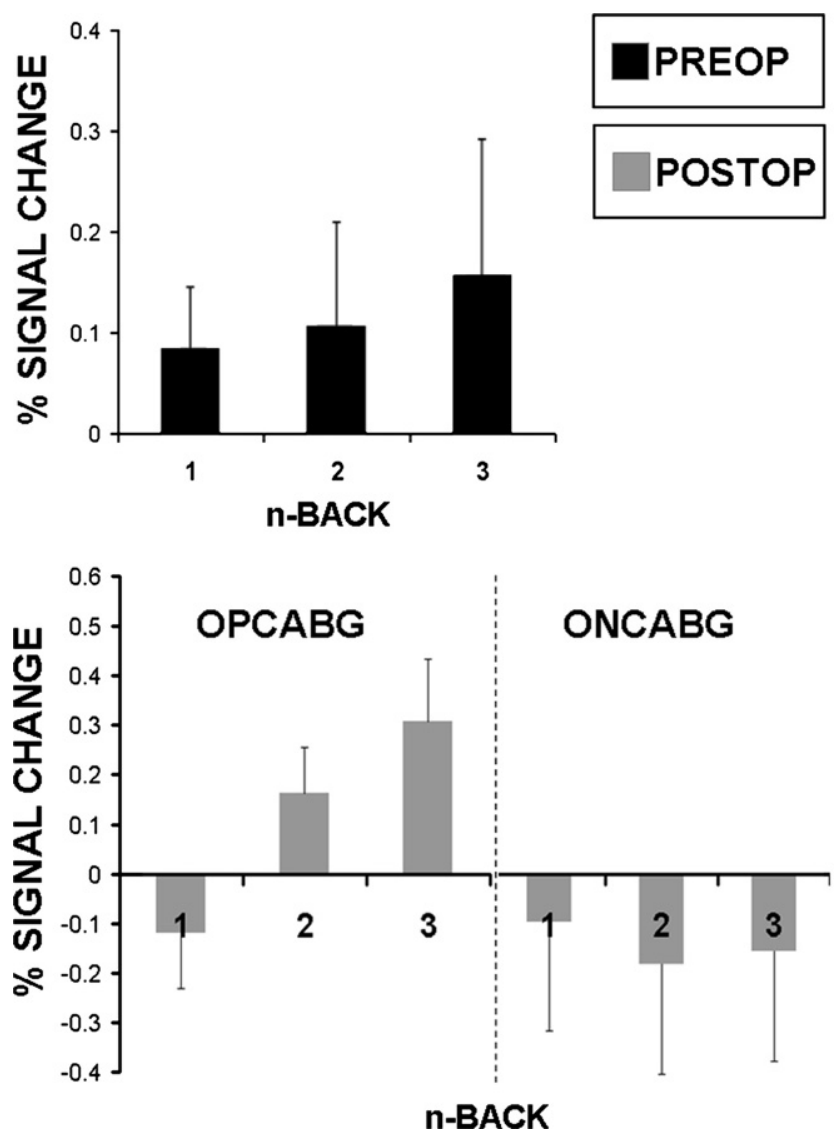

Figure 4. Percentage signal change in the prefrontal region before and after surgery.

additional insight into the pathophysiology of change and potential therapeutic approaches.

\section{Limitations}

It is important to consider the limitations in our study design and methodology. Although we did not randomize patients, this may be of less relevance, because the primary goal was simply to establish a proof of principle for this new approach. In any event, the patients were of a similar age and had similar ventricular function and risk status. In addition, the analyses were performed by 2 observers blinded to the nature of the surgical intervention.

Although changes in fMRI have been demonstrated in various pathologic conditions, it is not clear whether these changes are related to reduced neuronal activity and information processing or are an altered balance of excitatory and inhibitory inputs. When the changes are localized, as in our study, we believe they represent altered neuronal-hemodynamic coupling and therefore are a reflection of cerebral functional impairment.

At the start of this study, it was not our routine practice to use arterial line filtration. Although we now do (and filters were used in the last 5 patients in the ONCABG group), evidence supporting their use is still not compelling. Microemboli are abundant during CPB despite the use of $40-\mu \mathrm{m}$ arterial filtration, ${ }^{29}$ and leucocyte-depleting arterial line filters do not improve neurocognitive outcome. ${ }^{30}$ Our own data (unpublished observations) demonstrate no significant reductions in the number of microemboli detected during $\mathrm{CPB}$ with the use of $40-\mu \mathrm{m}$ arterial line filtration.

Our postoperative study was conducted at approximately 1 month. Longer-term follow-up is necessary to provide additional information testing for either resolution or progression of these changes.

\section{Conclusions}

On-pump surgery results in significant changes in postoperative brain function which correlate with intraoperative microembolic load. Functional MRI may prove to be more sensitive than neuropsychological testing in assessing perioperative cerebral injury and may offer additional insights both into its pathophysiology and into potential therapeutic approaches.

\section{References}

1. Taggart DP, Westaby S. Neurological and cognitive disorders after coronary artery bypass grafting. Curr Opin Cardiol. 2001;16:271-6.

2. van Dijk D, Keizer AM, Diephuis JC, Durand C, Vos LJ, Hijman R. Neurocognitive dysfunction after coronary artery bypass surgery: a systematic review. J Thorac Cardiovasc Surg. 2000;120:632-9.

3. Newman MF, Kirchner JL, Phillips-Bute B, Gaver V, Grocott H, Jones $\mathrm{RH}$, et al. Longitudinal assessment of neurocognitive function after coronary-artery bypass surgery. N Engl J Med. 2001;344:395-402.

4. Newman MF, Grocott HP, Mathew JP, White WD, Landolfo K, Reves JG, et al. Report of the substudy assessing the impact of neurocognitive function on quality of life 5 years after cardiac surgery. Stroke. 2001;32:2874-81.

5. Taggart DP, Browne SM, Wade DT, Halligan PW. Neuroprotection during cardiac surgery: a randomised trial of a platelet activating factor antagonist. Heart. 2003;89:897-900.

6. Abu-Omar Y, Cifelli A, Matthews PM, Taggart DP. The role of microembolisation in cerebral injury as defined by functional magnetic resonance imaging. Eur J Cardiothorac Surg. 2004;26:586-91.

7. Rombouts SA, van Swieten JC, Pijnenburg YA, Goekoop R, Barkhof F, Scheltens P. Loss of frontal fMRI activation in early frontotemporal dementia compared to early AD. Neurology. 2003;60:1904-8.

8. Abu-Omar Y, Balacumaraswami L, Pigott DW, Matthews PM, Taggart DP. Solid and gaseous cerebral microembolization during offpump, on-pump, and open cardiac surgery procedures. J Thorac Cardiovasc Surg. 2004;127:1759-65.

9. Brucher R, Russell D. Automatic online embolus detection and artifact rejection with the first multifrequency transcranial Doppler. Stroke. 2002;33:1969-74.

10. Russell D, Brucher R. Online automatic discrimination between solid and gaseous cerebral microemboli with the first multifrequency transcranial Doppler. Stroke. 2002;33:1975-80.

11. Braver TS, Cohen JD, Nystrom LE, Jonides J, Smith EE, Noll DC. A parametric study of prefrontal cortex involvement in human working memory. Neuroimage. 1997;5:49-62.

12. Jezzard P, Matthews PM, Smith SM. Functional MRI: an introduction to methods. Oxford: Oxford University Press; 2001.

13. Jenkinson M, Smith S. A global optimisation method for robust affine registration of brain images. Med Image Anal. 2001;5:143-56. 
14. Jenkinson M, Bannister P, Brady M, Smith S. Improved optimization for the robust and accurate linear registration and motion correction of brain images. Neuroimage. 2002;17:825-41.

15. Honey GD, Fu CH, Kim J, Brammer MJ, Croudace TJ, Suckling J, et al. Effects of verbal working memory load on corticocortical connectivity modeled by path analysis of functional magnetic resonance imaging data. Neuroimage. 2002;17:573-82.

16. Smith EE, Jonides J, Marshuetz C, Koeppe RA. Components of verbal working memory: evidence from neuroimaging. Proc Natl Acad Sci $U$ S A. 1998;95:876-82.

17. Machulda MM, Ward HA, Borowski B, Gunter JL, Cha RH, O’Brien $\mathrm{PC}$, et al. Comparison of memory fMRI response among normal, MCI, and Alzheimer's patients. Neurology. 2003;61:500-6.

18. Logothetis NK. The underpinnings of the BOLD functional magnetic resonance imaging signal. J Neurosci. 2003;23:3963-71.

19. Taggart D. Off-pump surgery and cerebral injury. J Thorac Cardiovasc Surg. 2004;127:7-9.

20. Wilkinson D, Halligan P. The relevance of behavioural measures for functional-imaging studies of cognition. Nat Rev Neurosci. 2004;5: 67-73.

21. Johansen-Berg H, Rushworth MF, Bogdanovic MD, Kischka U, Wimalaratna S, Matthews PM. The role of ipsilateral premotor cortex in hand movement after stroke. Proc Natl Acad Sci U S A. 2002;99: 14518-23.

22. Reddy H, Narayanan S, Woolrich M, Mitsumori T, Lapierre Y, Arnold $\mathrm{DL}$, et al. Functional brain reorganization for hand movement in patients with multiple sclerosis: defining distinct effects of injury and disability. Brain. 2002;125:2646-57.

23. Pugsley W, Klinger L, Paschalis C, Treasure T, Harrison M, Newman $\mathrm{S}$. The impact of microemboli during cardiopulmonary bypass on neuropsychological functioning. Stroke. 1994;25:1393-9.

24. Stump DA, Kon NA, Rogers AT, Hammon JW. Emboli and neuropsychological outcome following cardiopulmonary bypass. Echocardiography. 1996;13:555-8.

25. Sylivris S, Levi C, Matalanis G, Rosalion A, Buxton BF, Mitchell A, et al. Pattern and significance of cerebral microemboli during coronary artery bypass grafting. Ann Thorac Surg. 1998;66:1674-8.

26. Brown WR, Moody DM, Challa VR, Stump DA, Hammon JW. Longer duration of cardiopulmonary bypass is associated with greater numbers of cerebral microemboli. Stroke. 2000;31:707-13.

27. Brooker RF, Brown WR, Moody DM, Hammon JW Jr, Reboussin DM, Deal DD, et al. Cardiotomy suction: a major source of brain lipid emboli during cardiopulmonary bypass. Ann Thorac Surg. 1998;65: 1651-5.

28. Abu-Omar Y, Taggart DP. Off-pump coronary artery bypass grafting. Lancet. 2002;360:327-30.

29. Fearn SJ, Pole R, Burgess M, Ray SG, Hooper TL, McCollum CN. Cerebral embolisation during modern cardiopulmonary bypass. Eur J Cardiothorac Surg. 2001;20:1163-7.

30. Whitaker DC, Newman SP, Stygall J, Hope-Wynne C, Harrison MJ, Walesby RK. The effect of leucocyte-depleting arterial line filters on cerebral microemboli and neuropsychological outcome following coronary artery bypass surgery. Eur J Cardiothorac Surg. 2004;25:267-74. 